\title{
The longevity assurance homologue of yeast lag1 (Lass) gene family (Review)
}

\author{
ANDREAS TEUFEL ${ }^{1}$, THORSTEN MAASS ${ }^{1}$, PETER R. GALLE $^{1}$ and NASIR MALIK ${ }^{2}$ \\ ${ }^{1}$ Department of Medicine, Johannes Gutenberg University, Mainz, Germany; ${ }^{2}$ Cellular Neurobiology \\ Branch, National Institute on Drug Abuse, National Institutes of Health, Baltimore, MD, USA
}

Received June 13, 2008; Accepted August 26, 2008

DOI: 10.3892/ijmm_00000110

\begin{abstract}
The Lass gene family contains a group of highly conserved genes that are found in eukaryotic species. The founding member, lag1, was discovered in a screen for yeast longevity genes. Subsequently, lagl homologs were discovered in other organisms including six mammalian paralogs. All Lass genes encode a highly conserved Lag1 domain and many also have an additional Hox domain. Lass proteins are ceramide synthases and therefore are critical for ceramide biosynthesis. Ceramide synthase is also a critical enzyme in the sphingolipid biosynthetic pathway. As ceramide and sphingolipids are key intermediates in diverse cellular processes such as cell growth, apoptosis, and stress response and may also play a role in cancer development, the function of Lass proteins is of great interest. In this review, we summarize the state of knowledge regarding Lass protein structure, biological function, and their emerging role in cancer development.
\end{abstract}

\section{Contents}

1. Yeast lagl and lac1

2. Non-yeast Lass homologues

3. Sequence and structure of Lass proteins

4. Function of non-yeast Lass proteins

5. Lass gene family in cancer development, a potential target for cancer therapy?

6. The Lass protein family: Unresolved questions

\section{Yeast lag1 and lac1}

The founding member of the Lass gene family was discovered in a yeast screen for genes that were preferentially expressed

Correspondence to: Dr Andreas Teufel, Department of Internal Medicine I, Johannes Gutenberg University, Building 605, Langenbeckstr. 1, 55101 Mainz, Germany

E-mail: teufel@uni-mainz.de

Key words: longevity, homeodomain, ceramide, cancer, apoptosis, stress response in younger cells (1). The gene was named longevity assurance gene $(\operatorname{lag} 1)$, and its deletion increased the replicative capacity of yeast cells hence lagl became the first longevity gene to be identified in any species. A later study revealed that the effect of lagl is more complex with moderate expression increasing and high expression reducing life span. Two groups utilized a GenBank database search to identify a yeast paralog of lag 1 which was named lacl. The lag1 and lac1 proteins were shown to be multipass ER membrane proteins (2-4). lac1 lag 1 mutants were either inviable or had severe growth defects depending on the strain of cells used (3). The phenotype in the growth defective strain resulted from an inability to sustain ER-to-Golgi transport of GPI-anchored proteins. As this transport requires sphingolipid synthesis it was speculated that this pathway might be defective in lag1 lacl mutants. Additional biochemical studies confirmed this hypothesis and identified the synthesis of ceramide, which is a key intermediary in sphingolipid biosynthesis, as the specific defect in lagl lacl mutants (Fig. 1) $(5,6)$. Formal confirmation that lagl and lacl were components of the yeast ceramide synthase came when they were isolated to near homogeneity and co-purified with a fumonisin B1 (FB1) sensitive acyl-CoAdependent ceramide synthase activity (6). FB1 is a compound produced by a fungal pathogen that specifically inhibits ceramide synthase and fatty acids are required for ceramide synthase catalyzed synthesis of ceramide. A third component of the lag1/lac1 complex, lip1, was identified by mass spectrometry. Lip1 is a single membrane spanning ER protein that is also required for FB1 sensitive, acyl-CoA-dependent ceramide synthesis (7). This series of experiments established that the yeast ceramide synthase complex consisted of at least a lag1/lac1/lip1 complex.

\section{Non-yeast Lass homologues}

GenBank database searches with the lag1 and lac1 sequences uncovered homologues in worms, mice, and humans $(4,8,9)$. These proteins were more similar to lag 1 than lac 1 with a particularly highly conserved stretch of 52 amino acids that was termed the Lag1 domain (3). As more family members have been identified, the motif has been extended to 200 amino acids (5). The only other proteins with sequence similarity to lag1 are translocation chain associated membrane proteins (TRAM) but they lack the Lag1 domain. The evolutionary conservation between yeast lag 1 and lac 1 and their vertebrate 


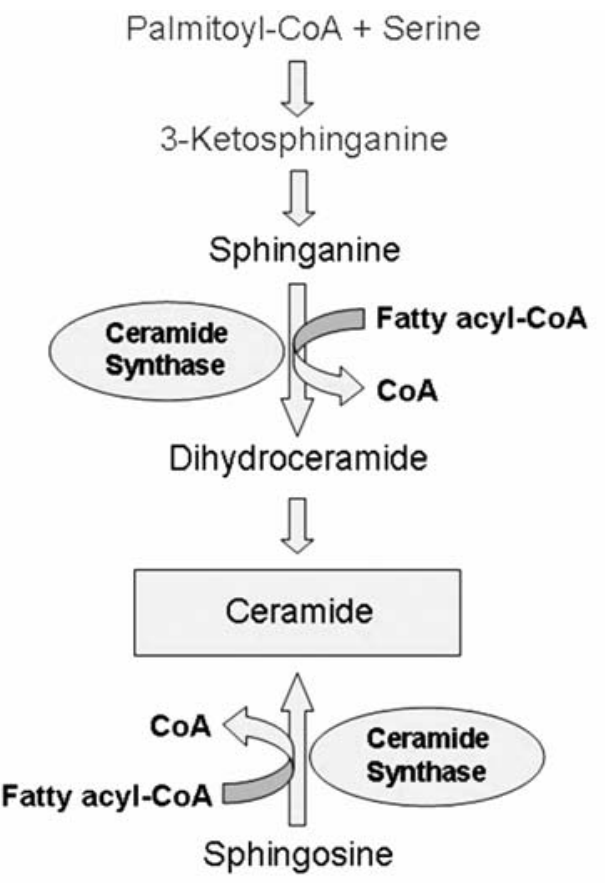

Figure 1. Schematic drawing of ceramide synthesis. Ceramide is composed of a sphingoid long chain base to which a fatty acid is attached via an amide bond. The attached fatty acids may vary with respect to their length between C14 and C32.

and invertebrate counterparts, but not TRAM, was dramatically shown when $C$. elegans and human lag homologs rescued the phenotype of yeast lagl lacl mutants (3). The name of the non-yeast lag genes was later changed to Lass (longevity assured homologue of yeast $\operatorname{lag} 1$ ). With the availability of complete genome sequences for many organisms it was found that all eukaryotes possess Lass genes. Interestingly, all non-Lass1 vertebrate Lass proteins contain a homeodomain motif which is normally found in nuclear transcriptional regulators $(8,10)$.

\section{Sequence and structure of Lass proteins}

The Lag domain. The family of Lass proteins is characterized by a conserved lag1 domain, located at the C-terminal part of the protein [Pfam identifier: PF03798, $(5,9)]$. The Lag1 domain contains 200 amino acids, with human Lass2, 3, 4, 5, and 6 lag1 domains containing 198 or 199 amino acids and the Lass1 Lag1 domain being somewhat longer with 213 amino acids. When aligning the lag 1 domains of all six human Lass homologues using the ClustalW algorithm (11), only $28 \%$ of all amino acids are at least $80 \%$ conserved among the homologues, and only $15 \%$ are completely conserved (Fig. 2). However, this comparably low conservation is enough to ensure ceramide synthase function for all members of the Lass family and may in contrast be the reason why these proteins promote ceramide synthesis with distinct fatty acid specificity for each member (discussed in a later section). Recently, targeted mutation experiments demonstrated that some of the conserved amino acids within the Lag1 domain were essential for ceramide synthesis (H182, H183, L189, D210, D213, L216), whereas others did not contribute to the biochemical process (S193, K220) (12).
This protein stretch containing the Lag1 domain has been described more broadly as part of the Tram-Lag-CLN8 (TLC) domain. The TLC domain is currently known to be shared by a group of 16 proteins, among them all Lass genes (Fig. 3). However, although TRAM has been identified to be structurally related to the Lag1 domain in yeast, the failure of TRAM proteins to complement the yeast lagl lacl deficiency suggests that other TLC family members do not have a ceramide synthase activity $(3,5)$. In addition, aligning all members of the TLC group, including all Lass proteins, the subgroup of Lass proteins is clearly distinct from other group members (Fig. 3). From the protein sequence alignments, it seems clear that the group of Lass genes although structurally related to TLC domain containing proteins comprises a distinct group of genes, both structurally and functionally. Alignment of all Lass orthologue proteins using ClustalW at EBI. The alignment clearly demonstrates the close relationship between Lass5 and 6 as well as Lass2, 3, and 4 (Fig. 4).

The homeodomain. All vertebrate Lass proteins except Lass1 contain a homeodomain. The Lass homeodomain is highly degenerate as only the very C-terminal 12 amino acids of the homeodomain, as recognized by SMART functional domain identification algorithm, are highly conserved among all members of the Lass family. By contrast most of the $\mathrm{N}$ terminus is highly variable. However, this degree of conservation is sufficient for it to be classified as a homeodomain by several motif-detection algorithms $(8,13)$.

Homeodomain-containing transcriptional regulators often operate differential genetic programs along the anteriorposterior axis of animal bodies (14). The domain binds DNA through a helix-turn-helix (HTH) structure. Thus, these proteins predominantly function as transcription factors important not only in embryonic but also cancer development (15). To date, there is no evidence of a transcriptional regulator function of Lass genes. The homeodomain must not be essential to ceramide synthase function as yeast lagl and lacl and human Lass1 both have ceramide synthase activity. A recent study demonstrated that for human Lass5 and 6 most of the homeodomain was dispensable for ceramide synthase activity but a 12 amino acid stretch of amino acids flanking the TLC and homeodomains is critical for ceramide synthase activity (16). This stretch includes the final eight amino acids of the homeodomain. It is still possible that the Lass proteins containing a homeodomain may have a novel function in addition to ceramide synthase activity in which the entire homeodomain plays a critical role.

Finally, it should be noted that all six genes in human (except, to date human Lass3) encode at least two isoforms, one of which lacks the homeodomain and part of the TLC domain; the relevance of these short isoforms is currently unknown but may imply similar mechanisms of transcriptional regulation and potentially unique isoform specific functions (17).

Transmembrane topology of Lass proteins. The transmembrane topology of yeast lac1 and lag1 was empirically determined by insertion of glycosylation sites and analysis of these modified proteins in yeast. This analysis revealed that lac1 and lag1 possess eight transmembrane spanning domains 
hLass5/1-392

hLass6/1-384

hLASS 2/1-380

hLass $3 / 1-383$

hLASS4/1-394

hLass $1 / 1-350$

hLass 5/1-392

hLass6/1-384

hLASS2/1-380

hLass $3 / 1-383$

hLASS $4 / 1-394$

hLass $1 / 1-350$

hLass 5/1-392

hLass 6/1-384

hLASS2/1-380

hLass $3 / 1-383$

hLASS4/1-394

hLass $1 / 1-350$

hLass 5/1-392

hLass6/1-384

hLASS2/1-380

hLass $3 / 1-383$

hLASS4/1-394

hLass $1 / 1-350$

hLass 5/1-392

hLass6/1-384

hLASS2/1-380

hLass 3/1-383

hLASS4/1-394

hLass 1/1-350

hLass 5/1-392

hLass6/1-384

hLASS2/1-380

hLass $3 / 1-383$

hLASS4/1-394

hLass $1 / 1-350$

hLass 5/1-392

hLass6/1-384

hLASS2/1-380

hLass $3 / 1-383$

hLASS4/1-394

hLass $1 / 1-350$
MATAAQGPLS LLWGW LWSERFWL PENV SWADLE GPADGYGYPRGRHILSV FPLAAGIFFV ------MA GILAW FUNERFUL PHNVTWADLKNTEEAT-FPQAED LYLA FP LAFC IFIV $-----M L Q T L Y D Y$ FWWERL UL PVNL TWADLE -DRDGRVYAKA SD LYITLPLALL FLIV --- --MFUTFKEW FULERFUL PPTI KWSDLE -DHDG LVFVKP SHLYVT I PYAFL LLII - -- --MLS SFNEW FWQDRFUL P PNVTWTELE -DRDGRVYPHP QD LLAA LPLALVLLAM -MAAA GPAAG PTGPEP ---------MPSY

\section{Homeodomain}

RLLFERFIAK PCALC IGIEDS GPYQAQ PNAI LEKVFIS ITKYPD KKRLEG LSKQLD WNVR RLIFERFVAK PCAIA LNIOANGPQIAP PNAI LEKVFTA ITKHPDEKRLEG LSKOLD WDVR RYFFELYVAT PLAAL LNIKEKTRLRAP PNATLE HFYLTSGKQPKQVEVEL LSRQSGLSGR RRVFEKFVAS PLAKS FGIKETVR-KVT PNTVLENFFKHSTRQPL QTDIYG LAKKCN LTER RLAFERFIGL PLSRU LGVRDQTRRQVK PNATLEKHFLTEGHRPKEPQLSL LAAQCGLTLQ $\overline{A Q L V}$ ORGUGSALAAARGCTDC GUGLARRGLAEHAHLAP PELLLLALGALGWTALRSAATA

KIQCUFRHRRNQDKP PTLTKFCESMUR FTFYLC IFCYG IRFLU- - SSPUFUDIRQC WHNY SIQRUFRQRRNQEKP STLTRFCESMUR FSFYLYVFTYGVRFLK - KTPUL WNTRHC WYNY QVERUFRRRRNQDRP SLLKKFREASUR FTFYLIAFIAGMAVIV- -DKPUFYDMKKVWEGY QVERUFRSRRNQERP SRLKKF QEACUR FAFYLM ITVAGIAFLY- -DKPUL YDL WEVWNGY QTQRUFRRRRNQDRP QLTKKF CEASWR FLFYLS SFVGG LSVLY- -HESUL WAPVMC WDRY RLFR PLAKRC CLQ-PRDAAKM PESAUKFLFYLGSUSYSAYLLFGTDYPFFHDP PSVFYUU
PF-0 PLSSGL YHYYIMELAFY - USLMF SQFTD I KRKDF LIMFVHHLVTIGLISFSY IMMM PY-0 PLTTDLHYYYI LELSFY-WSLMF SQFTDI KRKD FGIMFLHHLVSIF L IT SYVMN PI-0STIPSQYWYYM IELSFY -WSLLFSIASDVKRKD FKEQIIHHVATII LISFSWFANY PK-0 PLLPSQYWYYI LEMSFY - WSL LFRLGFDVKRKDF LAHIIHHLAAIS LMSF SWCANY PN-QTLKPSL YWWYL LELGFY -LSLLIRL P FDVKRKDFKEQVI HHFVAVI LMT F S YANL TPGMAVPRD I AAAYL LQGSFY GHSIYA TLYMT TWRKD VVMLLHHVVTLI LIVS SYAFRY

\section{LAG1 Domain}

VRVGTLIMCLHDVSDFLLEAARLANYAK-------YQRLCDT LFVIFSAVFFVTRLGI ARVGTLVLCLHD SAD ALLEAAKMANYAK--------FQKMCDL LFVMFAVVFITTRLGI IRAGTLIMALHD SSD YLLESA NMFNYA G-------WKNTCNN IFIVFA IVFIITRLVI IRSGTLVMIVHDVAD IULESARMFSYA G-------WTOTCNT LFFIFSTIFFISRLIV LRIGSLVLLLHD SSD YLLEAC KMVNYMQ-------YQQVCDA LFLIFS FVFFYTRLVL HNVG ILVLFLHDISDVOLEFTKLNIYFKSRGGS YHRLHALAADL GCLSFGFSWFUFRLYW

YPFU ILNTTL FESWE I IGPYA SWULLNGLLLTL QLLHV IUSYLI ARIALKALIRGKVSKD FPLUVLNTTL FESWE IVGPYP SWWVFN LLLLVQGLNC FWSYLI VKIACKAVSRGKVSKD LP FU ILHCTL VYPLE L YPAFF GYYFFN SMMGVL QLLHI FUAYLI LRMAHKFIT-GKLVED FPFUILYCTL ILPMYHLEPFF SYIFLN LLMIL QVLHL YWGYY LKMLNRCIF-NIKSIQD FPTQILYTTYYESISNRGPFF GYYFFNGLLMLLQLLHV FUSCLI LRMLYS FMIKKGQMIEKD FPLKVLYATSHCSLRTVPDIP FYFFFN ALLLLLTLMNL YWFLYIVAFAAKVLTGQVHELK

Figure 2. Alignment of the Lag1 domains of the human Lass homologues using ClustalW at EBI. Homeo- and Lag1-domain are in grey, the conserved amino acids within the Lag1 domain essential for ceramide synthesis are black. The transmembrane domains are underlined.

$(5,18)$. When an alignment of the protein sequences of Lass 1 family member is made it appears that all non-yeast Lass (except Lass1) proteins have seven transmembrane domains (TMD) with the putative second TMD of the yeast proteins being absent in all other family members (Fig. 2). The TLC domain includes TMDs 2-8 of yeast Lag 1 and Lac1 and TMDs 2-7 of the non-yeast Lass proteins. Most of the Lag1 domain encompasses TMD5-6 (yeast), TMD4-5 (non-yeast) with several amino acids in the ER lumen between these TMDs and some just $\mathrm{N}$ - and C-terminal of the first and 


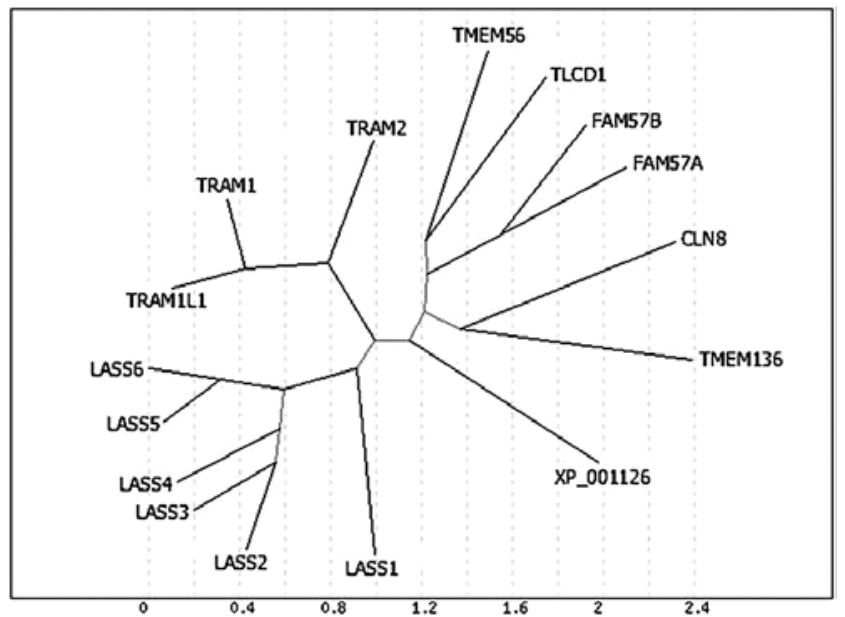

Figure 3. Alignment of all Lag1 domain containing proteins using ClustalW at EBI. The alignment clearly demonstrates the Lass genes being a distinct subgroup with Lass1 being more distantly related to the remaining family members as it does not contain a Hox domain (Ref-Protein IDs: Lass 1: NP_067090, Lass2: NP_071358, Lass3: NP_849164, Lass4: NP_078828, Lass5: NP_671723, Lass6: NP_982288, TMEM56: NP_689700, TRAM1L1: NP_689615, TRAM2: NP_036420, CLN8: NP_001029233, TRAM1: NP_055109, TMEM136: NP_777586, FAM57B: NP_113666, FAM57A: NP_079068, XP_001126126, TLCD1: NP_612472).

second of these TMDs. The homeodomain faces the cytoplasmic side in between TMDs 1 and 2 of the vertebrate nonLass1 proteins $(5,18)$.
Subcellular localization of Lass proteins. As the ER is the site of ceramide synthesis Lass proteins would be expected to localize to this organelle and in fact the subcellular localization of yeast lag1 was the ER membrane (3). Yeast lag1 is the only yeast Lass protein for which subcellular localization studies have been performed and its Lag1 contains a C-terminal ER retention signal. Human Lass1 and 2, and the three $C$. elegans Lass proteins also have a $\mathrm{C}$-terminal ER-retention signal and yeast lac1 has an N-terminal ER-retention motif. Although other Lass proteins lack an ER-retention motif such a motif is not required for ER localization and a recent study showed that Lass 5 which lacks a recognizable ER-retention motif does in fact localize to the ER (16).

\section{Function of non-yeast Lass proteins}

Since human Lass 1 rescued the yeast lagl lacl mutant it was reasonable to assume that Lass1 was a component of the mammalian ceramide synthase. The first study showing Lass1 had ceramide synthase activity in mammalian cells came from observations that when the human embryonic kidney 293T cell line is transfected with Lass 1 there is an FB1 sensitive, acyl-CoA-dependent increase in ceramide synthesis $(19,20)$. Interestingly, the increase in ceramide synthesis occurred only in the presence of C18 acyl-CoA chains. Thus, this result suggested fatty acid selectivity by Lass paralogs/ ceramide synthase in mammalian ceramide synthesis. Subsequently, similar results have been obtained for other members of the mammalian Lass protein family. Lass2

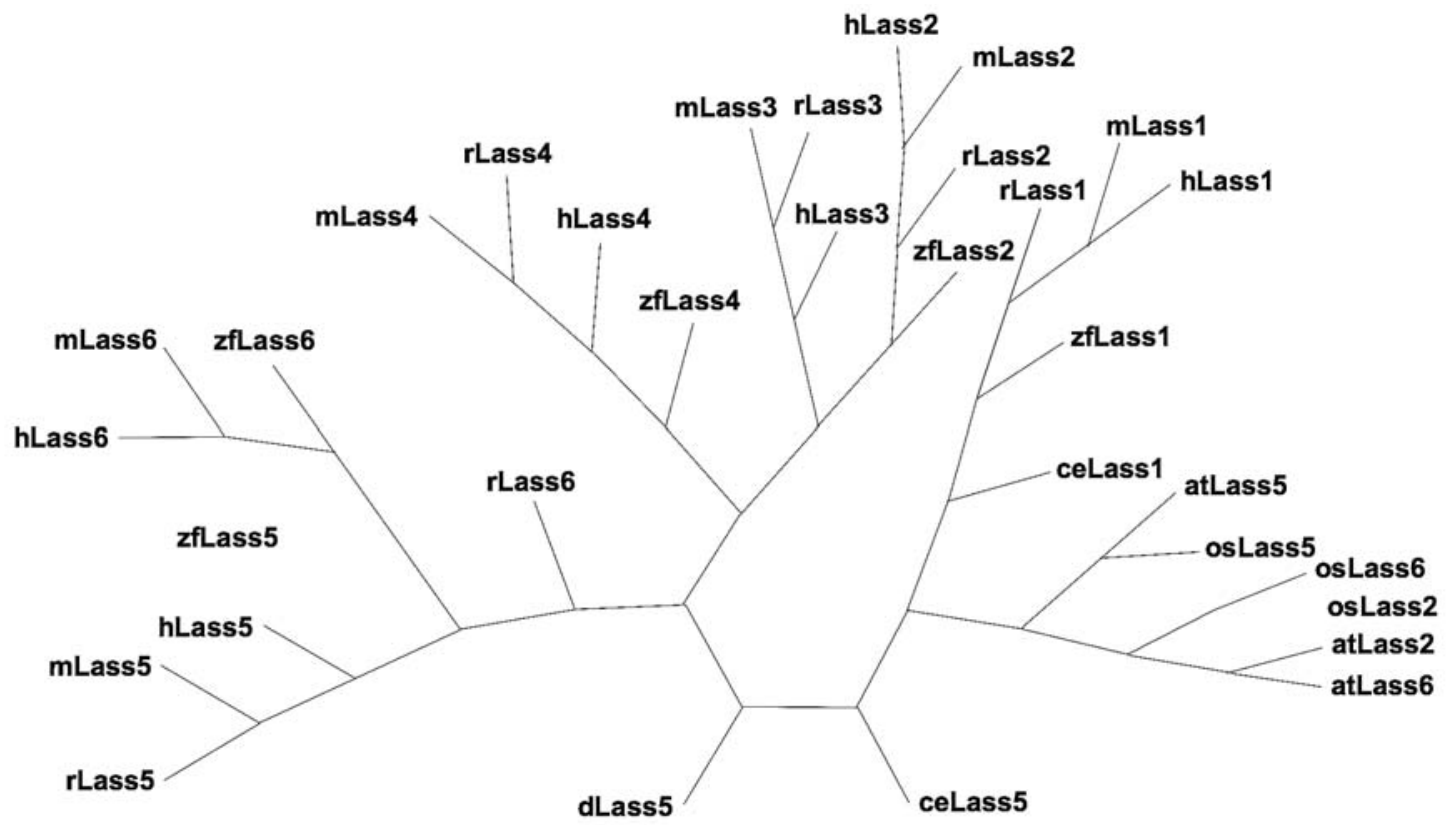

Figure 4. Alignment of all Lass orthologues proteins using ClustalW at EBI. The alignment clearly demonstrates the close relationship between Lass 5 and 6 as well as Lass2, 3, and 4. The plant orthologues Lass sequences ('at' for Arabidopsis thaliana and 'os' for Oryzias latipes) were more distant assembling in an individual group most closely associated to Lass1/lag1. (Ref-Protein IDs: hLass1: NP_067090.1, mLass1: NP_619588.1, rLass1: XP_224733.4, zfLass1: XP_696339.2, ceLass: 1NP 493403.1, hLass2: NP 071358.1, mLass2: NP 084065.1, rLass2: NP 001028872.1, zfLass2: NP 705957.1, atLass2: NP_001031037.1, osLass2: NP_001047244.1, hLass3: NP_849164.2, mLass3: XP_620510.2, rLass3: XP_574469.2, hLass4: NP_078828.1, mLass4: NP 080334.2, rLass4: XP 221796.4, zfLass4: NP 705956.1, hLass5: NP 671723.1, mLass5: NP 082291.1, rLass5: XP 345871.3, zfLass5: NP 955922.1, dLass5: NP_652526.1, ceLass5: NP_501459.1, atLass5: NP_188557.1, osLass5: NP_001049639.1, hLass6: NP_982288.1, mLass6: NP_766444.1, rLass6: XP_345364.3, zfLass6: XP_693283.2, atLass6: NP_566769.1, osLass6: NP_001047999.1). 
displayed a preference for long chain fatty acids (C22). Lass4 is involved in synthesis of longer ceramides such as C22:0and C24:0-ceramides. Lass 5 and 6 produce shorter ceramide species (C14:0- and C16:0-ceramides). By contrast, Lass3 exhibits relatively low substrate specificity toward fatty acylCoAs. Overexpression of Lass3 leads to increased levels of C18:0-, C22:0-, and C24:0-ceramides $(6,13,21,22)$.

Overexpression of the mammalian Lass proteins in yeast lagl lacl mutants can also result in some albeit a lower degree of specificity in regards to fatty acyl-CoA chain length. Lass 1 generates C26:0, Lass2 and 4 C24:0, but Lass5 generates equal amounts of C12:0, C14:0, C16:0, C18:0, and C18:1 (7,23). The lower degree of specificity may be the result of species differences in ceramide synthase activity between yeast and mammals. As has been mentioned earlier that the yeast ceramide synthase consists of a lag1/lac1/lip1 complex. It should be noted that lipl is found only in yeast and the expression of Lass5 in a lipl lacl lag1 yeast mutant rescues ceramide synthesis (7).

The only other species in which Lass function has been examined is the tomato (24). FB1 belongs to a cLass of natural toxins that are known as sphinanine-analog mycotoxins (SAMs). Alternaria alternata $f$. sp. lycopersici (AAL) is a pathogen that produces a toxin that is also a SAM. It was discovered that a homozygous mutation in a tomato Lass gene (Asc-1) that results in a stop codon in the second exon was responsible for sensitivity to AAL. Expression of the normal gene in mutant plants rescued AAL and FB1 sensitivity. FB1 sensitivity has been rescued by Lass in at least one other plant species, Nicotiana. It was established that Asc- 1 is a lag/ Lass ortholog when it partially rescued the growth defect in lag1 lac1 mutants (25).

\section{Lass gene family in cancer development, a potential target for cancer therapy?}

As Lass genes may have a function in longevity and one of the hallmarks of longevity is resistance to cancer, this family may be involved in cancer development. Additionally, ceramide signaling has previously been demonstrated to be essential to apoptosis, disturbance of which may result in cancer development. Thus, it seems reasonable to investigate the role of Lass family members in cancer development. At present little information is available in this area.

Lass2 expression displays an inhibitory effect on cell growth in hepatocellular carcinoma (HCC) cells. The number of colonies formed by Lass2-transformed HCC cells was reduced to $\sim 20 \%$ of that of the vector control (26). In addition, an essential role for Lass1 and C18-ceramide in the regulation of growth of head and neck squamous cell carcinoma (HNSCC) was shown. Lass1 was shown to inhibit cell growth (70-80\%), via modulation of telomerase activity and induction of apoptotic cell death by mitochondrial dysfunction (27).

Finally, Lass5 was found to play an essential role in the prevention of immune escape by cancer cells as a result of antigen presentation processing defects. Since elimination of tumor cells by cytotxic T-cells is triggered by MHC class Irestricted antigen presentation of the tumor cell, impairment of this antigen presentation is common in human cancers to evade an immune response (28-30). However, it was noted that a specific T-cell population is able to target these cells with impaired antigen presentation in part by presenting a Lass 5 fragment as an epitope. This study shed light on a possible effective application of Lass5 peptides in peptidebased vaccines for CTL-mediated targeting of processingdeficient tumors (31).

In general, Lass proteins have been demonstrated to regulate ceramide synthesis and substantial evidence is accumulating for ceramides and ceramide synthesis in general with respect to cancer therapy. Multiple chemotherapeutic agents such as doxorubicin, irinotecan or paclitaxel and many others were demonstrated to increase the ceramide levels in cells (32). This increase of ceramides have been attributed to a stimulation of the de novo synthesis of ceramides but also speculatively to a disrupted ceramide catabolism (33). With this observation, the next steps would obviously be to manipulate and increase ceramide levels in cancer cells to induce apoptosis or to enhance cancer chemotherapy. To date, multiple agents modulating the sphingolipid and in particular the ceramide metabolism have been reported. Also many of these agents have demonstrated promising results in tumor growth inhibition (reviewed in ref. 33). However, most of these data were collected in vitro and have yet to be confirmed in in vivo models and also be evaluated with respect to possible side effects potentially limiting their therapeutic use.

Besides manipulating key regulatory enzymes in ceramide synthesis, direct intracellular delivery of antimitogenic and proapoptotic ceramides demonstrated potential therapeutic use in cancer therapy. Stover and Kester (35) first reported to have successfully challenged the limitations of intracellular delivery of ceramides due to its inherent lipid hydrophobicity and physicochemical properties. Using ceramide-formulated liposomes for intracellular delivery a significant inhibition of MDA-MB-231 cell proliferation was demonstrated, proof of principle for sufficient intracellular ceramide delivery and potential chemotherapeutic use. Furthermore, they were able to successfully transfer these results into a murine in vivo cancer model and reported that the systemic i.v. delivery of C6-ceramide (C6) in a pegylated liposomal formulation significantly limited the growth of solid tumors in a syngenic $\mathrm{BALB} / \mathrm{c}$ mouse tumor model of breast adenocarcinoma (35). Evaluating ceramides in clinical cancer therapy in phase I studies evaluating the substance for toxicity and dose finding will therefore be of great interest and have yet to be performed.

Together, ceramides and therefore also ceramide synthases must be considered to be of high potential value in cancer therapy and substances regulating these family members have yet to be evaluated in clinical studies. A promising approach to regulate ceramide synthesis to achieve anti-cancer properties may come from regulating the Lass gene and protein family members. This approach has yet to be tested in in vivo models.

\section{The Lass protein family: Unresolved questions}

There are many questions remaining regarding vertebrate Lass proteins. These include the fundamental structure of the vertebrate ceramide synthase (Lass monomer, homodimer, heterodimer, other higher order structure with additional accessory proteins), loss and gain of function phenotypes in 
mouse and other model species, the potential association of Lass genes with human diseases as has been found for the TRAM containing protein CLN8 in the neurodegenerative disorder neuronal ceroid lipoofuscinase (36), and as mentioned above the possibility that Lass genes could be part of future anti-cancer therapies. Answers to these questions are likely to have far-reaching consequences for the treatments of the many diseases caused by deregulation of ceramide.

\section{References}

1. D'Mello NP, Childress AM, Franklin DS, Kale SP, Pinswasdi C and Jazwinski SM: Cloning and characterization of LAG1, a longevity-assurance gene in yeast. J Biol Chem 269: 1545115459, 1994.

2. Barz WP and Walter P: Two endoplasmic reticulum (ER) membrane proteins that facilitate ER-to-Golgi transport of glycosylphosphatidylinositol-anchored proteins. Mol Biol Cell 10: 1043-1059, 1999.

3. Jiang JC, Kirchman PA, Zagulski M, Hunt J and Jazwinski SM: Homologs of the yeast longevity gene LAG1 in Caenorhabditis elegans and human. Genome Res 8: 1259-1272, 1998.

4. Benson DA, Karsch-Mizrachi I, Lipman DJ, Ostell J and Wheeler DL: GenBank. Nucleic Acids Res 36: D25-D30, 2008.

5. Winter E and Ponting CP: TRAM, LAG1 and CLN8: members of a novel family of lipid-sensing domains? Trends Biochem Sci 27: 381-383, 2002 .

6. Venkataraman K, Riebeling C, Bodennec J, Riezman H, Allegood JC, Sullards MC, Merrill AH Jr, et al: Upstream of growth and differentiation factor 1 (uog1), a mammalian homolog of the yeast longevity assurance gene 1 (LAG1), regulates $\mathrm{N}$-stearoyl-sphinganine (C18-(dihydro)ceramide) synthesis in a fumonisin B1-independent manner in mammalian cells. J Biol Chem 277: 35642-35649, 2002

7. Vallee B and Riezman H: Lip1p: a novel subunit of acyl-CoA ceramide synthase. EMBO J 24: 730-741, 2005.

8. Venkataraman $\mathrm{K}$ and Futerman AH: Do longevity assurance genes containing Hox domains regulate cell development via ceramide synthesis? FEBS Lett 528: 3-4, 2002.

9. Sonnhammer EL, Eddy SR and Durbin R: Pfam: a comprehensive database of protein domain families based on seed alignments Proteins 28: 405-420, 1997.

10. Gehring WJ, Affolter M and Burglin T: Homeodomain proteins Annu Rev Biochem 63: 487-526, 1994

11. Thompson JD, Higgins DG and Gibson TJ: CLUSTAL W: improving the sensitivity of progressive multiple sequence alignment through sequence weighting, position-specific gap penalties and weight matrix choice. Nucleic Acids Res 22: 4673-4680, 1994.

12. Spassieva S, Seo JG, Jiang JC, Bielawski J, Alvarez-Vasquez F, Jazwinski SM, Hannun YA, et al: Necessary role for the Lag1p motif in (dihydro)ceramide synthase activity. J Biol Chem 281 : 33931-33938, 2006

13. Mizutani Y, Kihara A and Igarashi Y: Mammalian Lass6 and its related family members regulate synthesis of specific ceramides. Biochem J 390: 263-271, 2005.

14. Alonso CR: Hox proteins: sculpting body parts by activating localized cell death. Curr Biol 12: R776-R778, 2002

15. Nunes FD, de Almeida FC, Tucci R and de Sousa SC: Homeobox genes: a molecular link between development and cancer Pesqui Odontol Bras 17: 94-98, 2003.

16. Mesika A, Ben-Dor S, Laviad EL and Futerman AH: A new functional motif in Hox domain-containing ceramide synthases: identification of a novel region flanking the Hox and TLC domains essential for activity. J Biol Chem 282: 27366-27373, 2007
17. Pewzner-Jung Y, Ben-Dor S and Futerman AH: When do Lasses (longevity assurance genes) become CerS (ceramide synthases)?: Insights into the regulation of ceramide synthesis. J Biol Chem 281: 25001-25005, 2006.

18. Kageyama-Yahara $\mathrm{N}$ and Riezman $\mathrm{H}$ : Transmembrane topology of ceramide synthase in yeast. Biochem J 398: 585-593, 2006.

19. Soriano JM, Gonzalez L and Catala AI: Mechanism of action of sphingolipids and their metabolites in the toxicity of fumonisin B1. Prog Lipid Res 44: 345-356, 2005.

20. Lahiri S and Futerman AH: LASS5 is a bona fide dihydroceramide synthase that selectively utilizes palmitoyl-CoA as acyl donor. J Biol Chem 280: 33735-33738, 2005.

21. Guillas I, Kirchman PA, Chuard R, Pfefferli M, Jiang JC, Jazwinski SM and Conzelmann A: C26-CoA-dependent ceramide synthesis of Saccharomyces cerevisiae is operated by Lag1p and Lac1p. EMBO J 20: 2655-2665, 2001.

22. Riebeling C, Allegood JC, Wang E, Merrill AH Jr and Futerman AH: Two mammalian longevity assurance gene (LAG1) family members, trh1 and trh4, regulate dihydroceramide synthesis using different fatty acyl-CoA donors. J Biol Chem 278: 43452-43459, 2003

23. Schorling S, Vallee B, Barz WP, Riezman H and Oesterhelt D: Lag1p and Lac1p are essential for the Acyl-CoA-dependent ceramide synthase reaction in Saccharomyces cerevisae. Mol Biol Cell 12: 3417-3427, 2001.

24. Brandwagt BF, Mesbah LA, Takken FL, Laurent PL, Kneppers TJ, Hille J and Nijkamp HJ: A longevity assurance gene homolog of tomato mediates resistance to Alternaria alternata $f$. sp. lycopersici toxins and fumonisin B1. Proc Natl Acad Sci USA 97: 4961-4966, 2000.

25. Spassieva SD, Markham JE and Hille J: The plant disease resistance gene Asc-1 prevents disruption of sphingolipid metabolism during AAL-toxin-induced programmed cell death. Plant J 32: 561-572, 2002.

26. Pan H, Qin WX, Huo KK, Wan DF, Yu Y, Xu ZG, Hu QD, et al: Cloning, mapping, and characterization of a human homologue of the yeast longevity assurance gene LAG1. Genomics 77: 58-64, 2001.

27. Koybasi S, Senkal CE, Sundararaj K, Spassieva S, Bielawski J, Osta W, Day TA, et al: Defects in cell growth regulation by C18:0-ceramide and longevity assurance gene 1 in human head and neck squamous cell carcinomas. J Biol Chem 279: 44311-44319, 2004.

28. Hicklin DJ, Marincola FM and Ferrone S: HLA class I antigen downregulation in human cancers: T-cell immunotherapy revives an old story. Mol Med Today 5: 178-186, 1999.

29. Seliger B, Maeurer MJ and Ferrone S: TAP off - tumors on. Immunol Today 18: 292-299, 1997.

30. Marincola FM, Jaffee EM, Hicklin DJ and Ferrone S: Escape of human solid tumors from T-cell recognition: molecular mechanisms and functional significance. Adv Immunol 74: $181-273,2000$.

31. van Hall T, Wolpert EZ, van Veelen P, Laban S, van der Veer M, Roseboom M, Bres S, et al: Selective cytotoxic T-lymphocyte targeting of tumor immune escape variants. Nat Med 12: 417-424, 2006.

32. Senchenkov A, Litvak DA and Cabot MC: Targeting ceramide metabolism - a strategy for overcoming drug resistance. J Natl Cancer Inst 93: 347-357, 2001.

33. Reynolds CP, Maurer BJ and Kolesnick RN: Ceramide synthesis and metabolism as a target for cancer therapy. Cancer Lett 206: 169-180, 2004.

34. Modrak DE, Gold DV and Goldenberg DM: Sphingolipid targets in cancer therapy. Mol Cancer Ther 5: 200-208, 2006.

35. Stover $\mathrm{T}$ and Kester M: Liposomal delivery enhances shortchain ceramide-induced apoptosis of breast cancer cells. J Pharmacol Exp Ther 307: 468-475, 2003.

36. Kyttala A, Lahtinen U, Braulke T and Hofmann SL: Functional biology of the neuronal ceroid lipofuscinoses (NCL) proteins Biochim Biophys Acta 1762: 920-933, 2006. 